\title{
SELECCIÓN DE ESTADÍSTICOS PARA LA ESTIMACIÓN DE LA PRECISIÓN EXPERIMENTAL EN ENSAYOS DE MAÍZ ${ }^{1}$
}

\author{
Román Gordón-Mendoza², Ismael Camargo-Buitrago²
}

\begin{abstract}
RESUMEN
Selección de estadísticos para la estimación de la precisión experimental en ensayos de maíz. El objetivo de este estudio fue seleccionar el estadístico más óptimo para estimar la precisión del experimento, así como evaluar el estimado del coeficiente de variación utilizado frecuentemente como una medida para estimar la validez de los ensayos. Para el análisis se incluyeron 406 experimentos; realizados en la Estación Experimental El Ejido y campo de productores colaboradores de la Región de Azuero, Panamá, del período comprendido entre los años 2000-2013. A cada experimento se le calculó los distintos componentes de la varianza o cuadrados medios (CM), así como el coeficiente de variación (CV), repetitividad, rango, diferencia mínima significativa (DMS), error estándar (EE), coeficiente de determinación $\left(\mathrm{R}^{2}\right)$ de los tratamientos y residuo. Se encontró que el CV estuvo más relacionado que la repetitividad con la media general del experimento (índice de correlación de 0,57 vs 0,24), por lo que le resta robustez para indicar precisión experimental. El aumento del número de repeticiones de experimentos con igual CM del error redujo el EE y aumentó la precisión. La repetitividad del experimento estuvo altamente asociada a los $\mathrm{R}^{2}$ tanto de los tratamientos $(0,93)$ como del error $(0,87)$, lo contrario se presentó para el CV $(0,23$ y 0,23 , respectivamente). Al relacionar ambos estadísticos con el cociente DMS/Rango se encontró que la repetitividad estuvo altamente correlacionada $\left(\mathrm{R}^{2}=0,76\right)$ con este cociente, mientras que el CV presentó un $\mathrm{R}^{2}$ de 0,18 . Este resultado sugiere que la repetitividad es un mejor indicador que el CV, de buena precisión experimental.
\end{abstract}

Palabras clave: repetitividad, coeficiente de variación, cuadrado medio del error, error estándar de la diferencia.

\begin{abstract}
Statistical selection for estimating the accuracy in experimental corn trials. The objective of this study was to select the most optimal statistic to estimate the experimental accuracy and to assess the Coefficient of Variation often used to estimate the validity of experiments. For the analysis, 406 trials were included; from research conducted at El Ejido Experimental Station and at cornfields of contributing producers of Azuero Region, Panama, during 2000-2013. To each trial it was calculated the various components of variance or Mean Square (MS), Coefficient of Variation (CV), Repeatability, Rank, Least Significant Differences (LSD), Standard Error (SE), Coefficient of determination $\left(\mathrm{R}^{2}\right)$ of treatment and residual. We found that the $\mathrm{CV}$ is more related than repeatability with the overall mean of the experiment (correlation index of 0.57 vs. 0.24 ), thus being less robust to indicate experimental accuracy. Increasing the number of repetitions of experiments with the same MS Error reduced the SE and increased accuracy. The repeatability of the experiment was highly related to both treatments $\mathrm{R}^{2}(0.93)$ and the unexplained fraction model (0.87), in contrast with the $\mathrm{CV}(0.23$ and 0.23 , respectively). When repeatability and CV were linked to the DMS/Range ratio, it was found that repeatability is highly correlated $\left(\mathrm{R}^{2}\right.$ $=0.76$ ) with this ratio, while the $\mathrm{CV}$ provided an $\mathrm{R}^{2}$ of 0.18 . This result suggests that the repeatability is a better indicator than the $\mathrm{CV}$ for good experimental accuracy.
\end{abstract}

Keywords: repeatability, coefficient of variation, mean square error, standard error of difference.

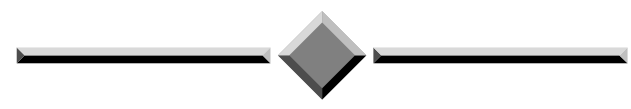

Recibido: 12 agosto, 2014. Aceptado: 14 de setiembre, 2014. Investigación financiada por el Proyecto Generación de Variedades e Híbridos de Maíz del Instituto de Investigación Agropecuaria de Panamá (IDIAP), Panamá.

2 Instituto de Investigación Agropecuaria de Panamá (IDIAP), Panamá. gordon.roman@gmail.com, camargo.ismael@gmail.com 


\section{INTRODUCCIÓN}

La calidad de los resultados experimentales es una preocupación de los investigadores al realizar sus trabajos de investigación, sean estos a nivel de campo o laboratorio, para decidir si los datos obtenidos son confiables y consecuentemente aceptar o no la validez de los mismos (Bowman y Rawlings, 1995). Para algunos investigadores el coeficiente de variación de Pearson es considerado fundamental como indicador de la calidad del experimento (RuízRamírez, 2010). Muchos científicos usan el coeficiente de variación $(\mathrm{CV})$ para aceptar o rechazar la validez de los experimentos (Bowman, 2001). El CV es la desviación estándar expresada como porcentaje de la media aritmética (Patel et al., 2001; Ruíz y Sánchez, 2006). Esto lo hace un coeficiente adimensional al estar conformado por una razón entre dos estadísticos que reflejan diferentes características de la población (Vásquez y Caballero, 2011).

Tradicionalmente el coeficiente de variación ha sido utilizado para decidir si un experimento es confiable o no. Esto último es criticado por varios autores debido a la relación que tiene este estadístico con la media ambiental (Barreto y Raun, 1990; Bowman y Watson, 1997; Taylor et al., 1999; Bowman, 2001), el CV deja de ser útil cuando la media se aproxima a cero (Silveira et al., 1986). No obstante, Gómez y Gómez (1984) y Patel et al. (2001) son más específicos al indicar que los $\mathrm{CV}$ varían considerablemente de acuerdo al tipo de experimento, indicando que los rangos aceptables deben ser entre 6 a $8 \%$ para evaluación de cultivares, 10 a $12 \%$ para fertilización y 13 a $15 \%$ para ensayos de evaluación de plaguicidas; por otro lado, Pimentel (1985) señala que normalmente en los ensayos agrícolas de campo los CV se consideran bajos cuando son inferiores a 10\%; medios de 10 a 20\%, altos cuando van de 20 a $30 \%$ y muy altos cuando son superiores a $30 \%$. Una gran cantidad de investigadores (Gómez y Gómez, 1984; Martínez, 1988; Patel et al., 2001), indican que si el valor del CV supera el 30\%, los datos deben ser descartados por la baja precisión que se tuvo.

Por otro lado, la heredabilidad o repetitividad $\mathrm{H}$ es un indicativo de la validez o utilidad de las pruebas de evaluación de genotipos, cuando $\mathrm{H}$ $=1$ significa que las diferencias observadas entre las medias genotípicas del ensayo son debido al efecto genético; mientras que $\mathrm{H}=0$ indica que las diferencias observadas son debido al error aleatorio o experimental (Yan y Holland, 2010).

El objetivo del estudio fue seleccionar el estadístico más óptimo para estimar la precisión del experimento, así como el de evaluar el estimado del coeficiente de variación utilizado frecuentemente como una medida para estimar la validez de los ensayos.

\section{MATERIALES Y MÉTODOS}

Para el desarrollo del presente estudio se utilizó la base datos de los ensayos realizados por el Proyecto de Maíz del Instituto de Investigación Agropecuaria de Panamá (IDIAP), del período comprendido entre los años 2000 a 2013. Estos ensayos consistieron de los experimentos recibidos del Centro Internacional de Mejoramiento de Maíz y Trigo (CIMMYT) y los experimentos de evaluaciones de cultivares del IDIAP. Los mismos se realizaron en campos de maíz de productores colaboradores del Proyecto, así como en la Estación Experimental El Ejido, ubicada en Los Santos, Panamá a una latitud de $7^{\circ} 54^{\prime}$ norte y $80^{\circ} 22^{\prime}$ longitud oeste.

En total se analizaron 406 experimentos, todos con el diseño Alfa Látice (Vargas et al., 2013). El número de tratamientos evaluados en estos ensayos fue variado; el número máximo de tratamientos evaluados en un ensayo fue de 150 , mientras que el ensayo con el menor número incluyó ocho entradas (Figura 1A). La base de datos que se analizó incluyó 234 ensayos de adaptación de materiales comerciales y semi-comerciales, sembrados fuera de la estación experimental y 172 ensayos de cultivares experimentales sembrados en la Estación. El material genético incluyó viveros de líneas endogámicas (L), híbridos simples (S), triples (T), dobles (D) y variedades sintéticas (V) (Figura 1B).

Los ensayos analizados tuvieron distintos número de repeticiones, agrupándose de la siguiente manera: 80 tenían dos repeticiones, 315 tuvieron tres, mientras que con cuatro repeticiones se analizaron once experimentos. Para este trabajo se tomó la variable rendimiento de grano ( $\mathrm{t} / \mathrm{ha}$ ) de todos los experimentos. En total la base de datos estuvo conformada por 9927 entradas del rendimiento de grano de todos los experimentos comprendidos en el estudio. 

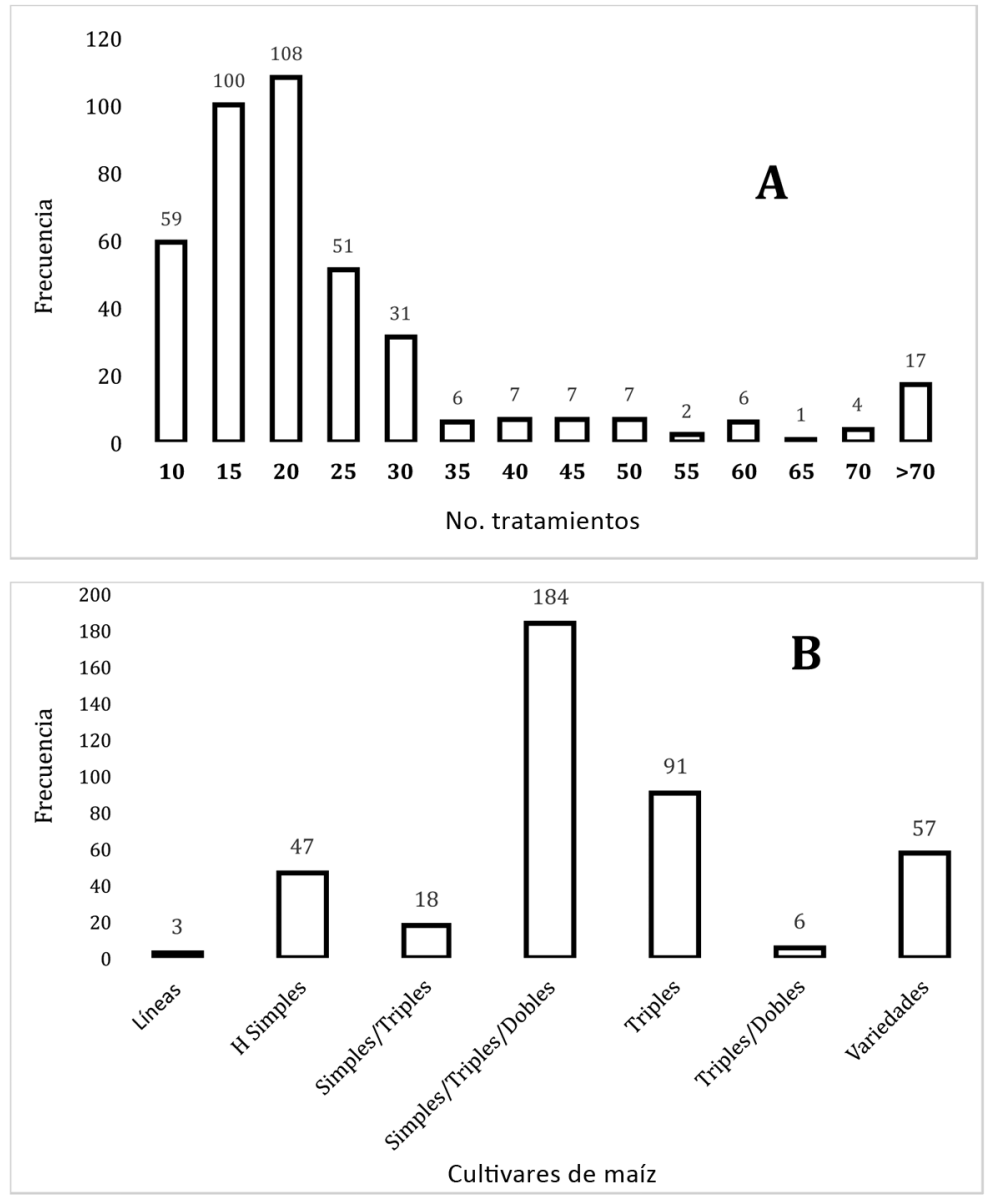

Figura 1. Distribución del número de tratamientos por ensayos (A) y líneas endogámicas, híbridos simples, triples, dobles y variedades sintéticas por ensayos (B) incluidos en la base experimental. Panamá, 2000-2013.

En cada experimento, a la variable rendimiento de grano se le calculó los distintos componentes de varianza del modelo lineal. Para esto se utilizó el método REML implementado en el procedimiento MIXED de SAS (Vargas et al., 2013). Adicional a las varianzas o cuadrados medios $(\mathrm{CM})$ del modelo $(\mathrm{CM}$ repetición, $\mathrm{CM}$ bloque $\mathrm{x}$ repetición, $\mathrm{CM}$ tratamientos y $\mathrm{CM}$ error), se calculó: el coeficiente de variación $(\mathrm{CV})$, repetitividad $\left(\mathrm{h}^{2}\right)$, rango, diferencia mínima significativa (DMS), error estándar (EE), coeficiente de determinación $\left(\mathrm{R}^{2}\right)$ de los tratamientos y $\mathrm{R}^{2}$ de la fracción no explicada por el modelo (residuo). Adicional a estos estadísticos se calculó la razón entre el DMS y el rango del experimento (D/R). Las fórmulas utilizadas para el cálculo de los estadísticos fueron las siguientes:

$$
\begin{array}{cc}
Y_{i j k}=\mu+\operatorname{Rep}_{i}+\text { Block }_{j}\left(\text { Rep }_{i}\right)+\text { Gen }_{k}+\varepsilon_{i j k} \\
C V=\frac{\sqrt{\text { CMError }}}{\bar{x}} * 100 & h^{2}=\frac{\delta_{\text {Trat }}^{2}}{\delta_{\text {Trat }}^{2}+\frac{\delta_{\text {Error }}^{2}}{\text { Norep }}} \\
\text { Rango }=\text { Máx }- \text { Min } & D M S_{5 \%}=t * S_{\bar{X}_{1}}-\bar{X}_{2} \\
R_{\text {No Expl }}^{2}=\frac{\text { SCError }}{\text { SCTotal }} & R_{\text {Trat }}^{2}=\frac{\text { SC Tratamientos }}{\text { SCTotal }}
\end{array}
$$




$$
S_{\bar{x}}=\sqrt{\frac{\text { CMError }}{\text { No reps }}} \quad \text { Indice } D / R=\frac{D M S}{R A N G O}
$$

Calculados todos los estadísticos por ensayo, se graficaron y correlacionaron tanto el CV como el $\mathrm{h}^{2}$ con los demás estadísticos calculados.

\section{RESULTADOS Y DISCUSIÓN}

El análisis general de los datos de rendimiento y el cálculo de los principales estadísticos descriptivos se presenta en el Cuadro 1. El resultado de este análisis indicó una media de 6,07 t/ha y varianza de 2654 t/ ha. Se elaboró un histograma con todos los datos y la hipótesis de normalidad fue significativa, indicando que los datos analizados tienen una distribución normal con media cero y varianza uno (Figura 2).

\section{Relación entre los estadísticos EE, CME, CV, h $\mathbf{h}^{2}$ y t/ha}

Al graficar el cuadrado medio del error (CME) contra el EE de cada ensayo, se observaron tres curvas bien demarcadas. Estas corresponden al número de

Cuadro 1. Estadísticas descriptivas de la data utilizada para el análisis de estimación de la precisión experimental. Panamá. 2000-2013.

\begin{tabular}{lc}
\hline Estadísticos calculados & Rendimiento \\
\hline Media & 6,07 \\
Error típico & 0,016 \\
Mediana & 6,13 \\
Moda & 5,31 \\
Desviación estándar & 1,629 \\
Varianza de la muestra & 2,654 \\
Curtosis & 0,55 \\
Coeficiente de asimetría & $-0,30$ \\
Rango & 12,90 \\
Mínimo & 0,18 \\
Máximo & 13,08 \\
No datos & 9927 \\
Años & 13 \\
Nivel de confianza $(95,0 \%)$ & 0,032 \\
\hline
\end{tabular}

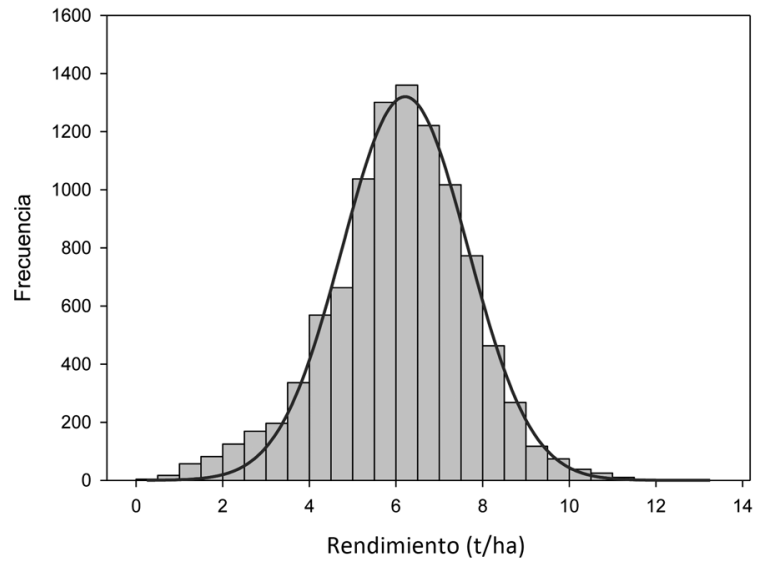

Figura 2. Histograma del rendimiento de grano de cada uno de los 406 experimentos analizados. Panamá 2000-2013.

repeticiones empleadas para la formación de cada uno de ellos. Al analizar este resultado, se observó que para un mismo valor del CME, el EE aumentó al pasar de la curva de tres a la de dos repeticiones. Esto implica que se tiene menos precisión en un ensayo con dos repeticiones, dado que el valor del DMS o cualquier otro estadístico para separar las medias es mayor. Esta misma relación se observó del pasar de cuatro a tres repeticiones, pero la ganancia en precisión fue más baja, dada la menor diferencia entre estas dos curvas (Figura 3), Gauch y Zobel (1996), encontraron

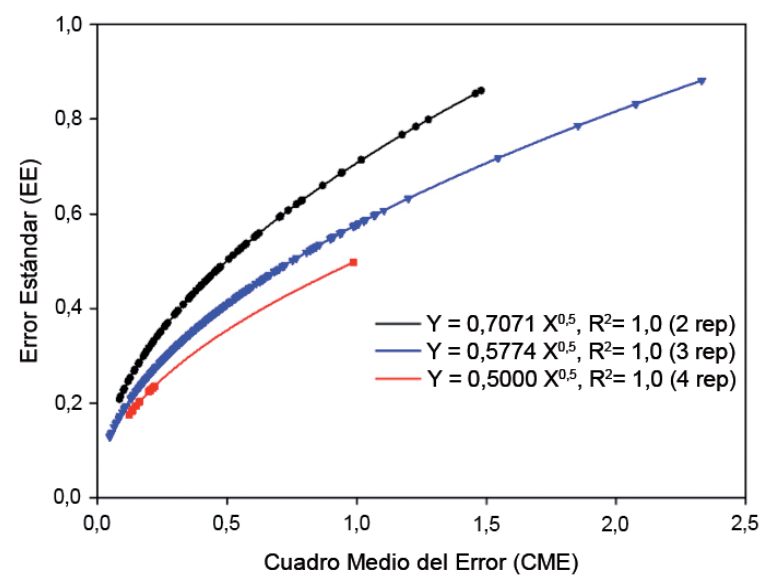

Figura 3. Relación entre el cuadrado medio del error y error estándar de la diferencia de dos medias para cada uno de los 406 experimentos analizados según número de repeticiones. Panamá. 2000-2013. 
que mayor número de repeticiones reduce el error experimental.

Cuando se relacionó tanto el $\mathrm{CV}$ como la $\mathrm{h}^{2}$ con la media experimental de cada ensayo (Figura 4), se encontró que el CV tuvo un coeficiente de correlación más alto (más del doble) que con el valor de la repetitividad $(0,57$ vs 0,24$)$. Resultados similares encontraron Barreto y Raun (1990), Bowman y Watson (1997) y Bowman (2001). Esta relación indica que por estar el CV altamente asociado con la media experimental de los ensayos realizados en zonas con elevado estrés, pueden presentar CV más altos que los mismos ensayos ubicados en áreas de alto potencial de rendimiento. Por el contrario, en la Figura 4B Rend
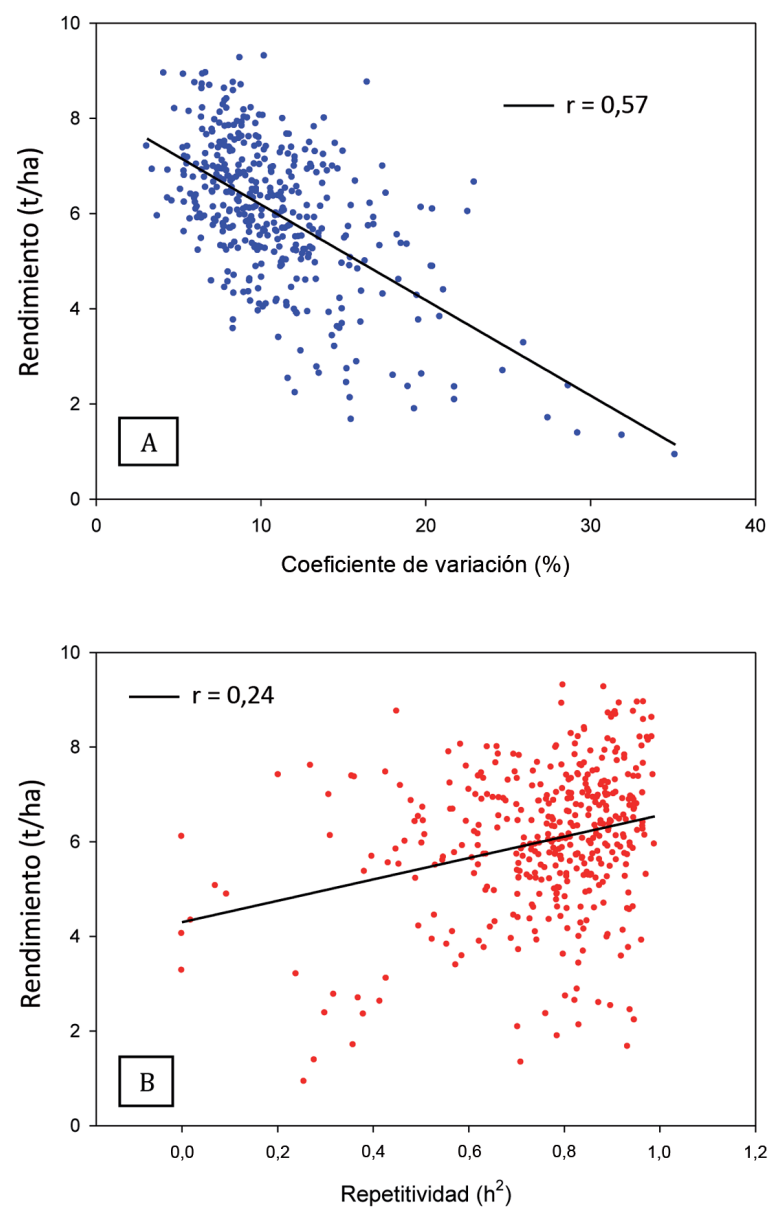

Figura 4. Relación del coeficiente de variación y la repetitividad para la media experimental de cada ensayo. Panamá. 2000-2013. $v s \mathrm{~h}^{2}$, la cercanía de la mayoría de los puntos al 1,0 sugiere que las diferencias observadas fueron debido a los efectos genotípicos (Yan y Holland, 2010).

Por otro lado, al relacionar el $\mathrm{CV}$ con $\mathrm{h}^{2}$ no se encontró una relación entre ambos estadísticos, al estimarse un coeficiente de correlación de 0,46. Resultados similares de poca relación entre estos dos parámetros fueron señalados por Yan y Holland (2010). No obstante, en la Figura 5 se presenta la tendencia de esta situación, siendo la misma inversa; es decir, que a mayor valor de repetitividad menor fue el coeficiente de variación. También se observa que valores de $\mathrm{h}^{2}$ mayores de 0,90 presentaron menor dispersión de $\mathrm{CV}$, posiblemente porque la variabilidad experimental se debió a efectos genéticos y una baja proporción de la variabilidad debido al error experimental que normalmente aumenta y dispersa el valor del CV.

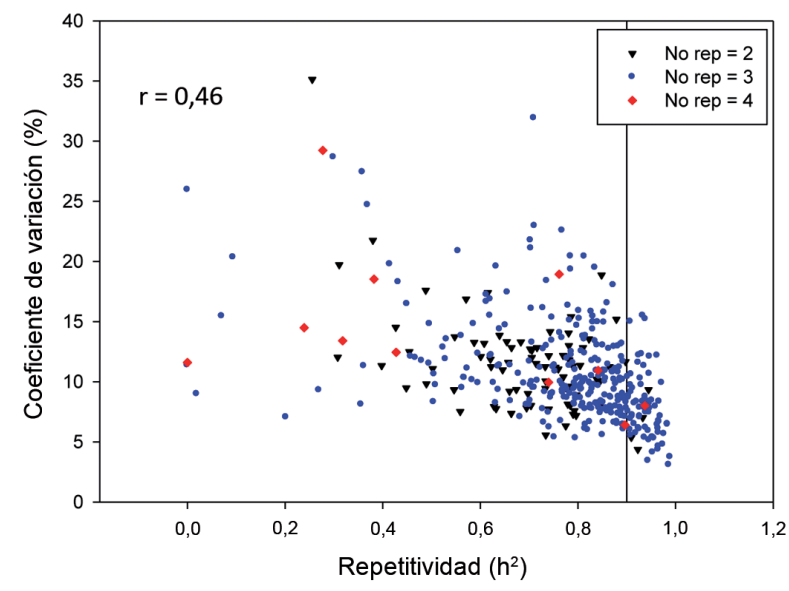

Figura 5. Relación entre el coeficiente de variación y los valores de repetitividad de los 406 experimentos analizados. Panamá. 2000-2013.

\section{Valoración de la precisión experimental}

Para estimar la precisión de los experimentos se relacionaron los estadísticos $\mathrm{CV}$ y $\mathrm{h}^{2}$ con los coeficientes de determinación del modelo $\left(\mathrm{R}^{2}{ }_{\text {Trat }} \mathrm{y}\right.$ $\mathrm{R}_{\text {No explicado }}^{2}$. Se graficaron ambos parámetros con el coeficiente de determinación de los tratamientos y se encontró que el CV presentó un $\mathrm{R}^{2}$ de $0,17,0,23$ y 0,52 para los ensayos con dos, tres y cuatro repeticiones, 
respectivamente (Figura 6A). Estos coeficientes son muy bajos con respecto a los valores encontrados en la relación del $\mathrm{R}^{2}$ de tratamiento $v s$ repetitividad cuyos coeficientes de determinación fueron de 0,99, 0,93 y 0,96 para estos mismos ensayos (Figura 6B). En resumen la relación del $\mathrm{h}^{2}$ con el $\mathrm{R}^{2}$ de los tratamientos resultó directa. Estos resultados indican que un valor alto de $\mathrm{h}^{2}$ (cercano a 1,0$)$ está muy correlacionado con un valor alto del $\mathrm{R}^{2}$ de tratamientos, interpretándose por consiguiente que la mayor variación del experimento se debió a la alta variabilidad entre los tratamientos evaluados (Yan y Holland, 2010). Por el contrario, un valor bajo del $\mathrm{h}^{2}$ sugiere que hubo una baja variabilidad entre los tratamientos o un alto valor del cuadrado medio del error, lo que indicaría un mal manejo del experimento (Martínez, 2005). Se encontró una diferencia entre las respuestas según el número
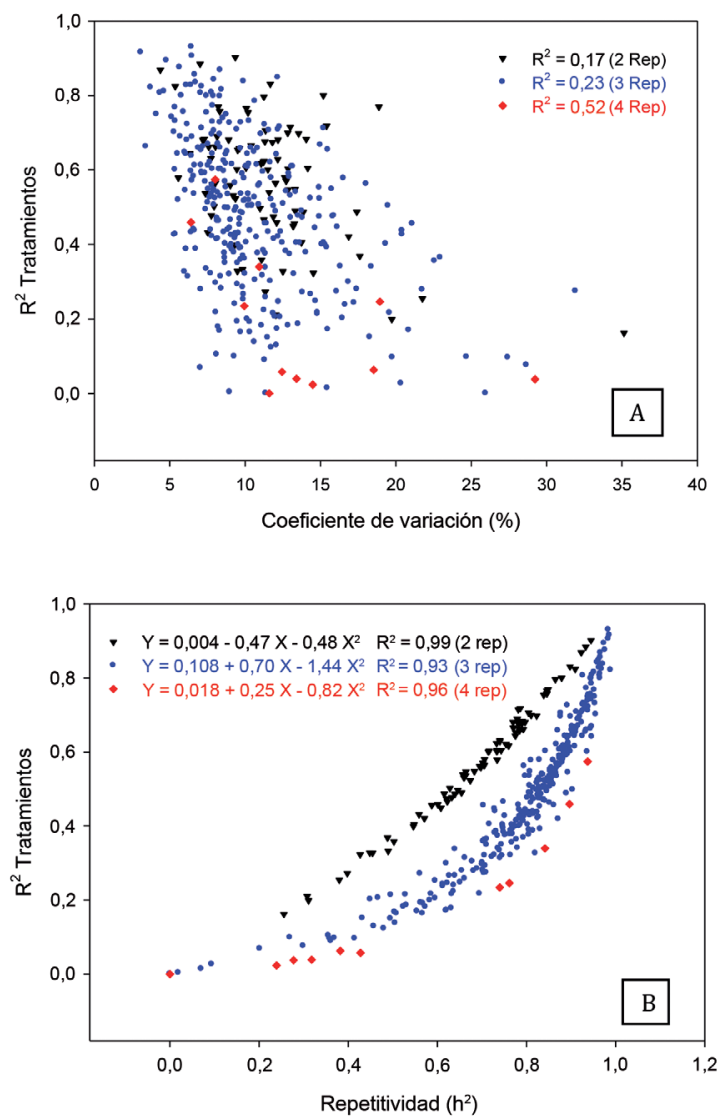

Figura 6. Relación entre el coeficiente de determinación de tratamientos con el coeficiente de variación y repetitividad, según el número de repeticiones de los experimentos. Panamá. 2000-2013. de repeticiones de los experimentos, en donde valores más bajos del $\mathrm{R}^{2}$ de tratamientos presentaron valores más altos de repetitividad.

Al analizar los estadísticos $\mathrm{CV}$ y $\mathrm{h}^{2}$, con el coeficiente de determinación de la fracción no explicada del modelo (residuo o $\mathrm{R}^{2}$ del error). Donde el coeficiente de variación no presentó ninguna relación con el error, este presentó un $\mathrm{R}^{2}$ de 0,17 , 0,23 y 0,652 para dos, tres y cuatro repeticiones, respectivamente (Figura 7A). Por el contrario, el coeficiente de repetitividad presentó altos valores de $\mathrm{R}^{2}$ en las ecuaciones que relacionaron este estadístico con el $\mathrm{R}^{2}$ del error con valores de 0,98, 0,87 y
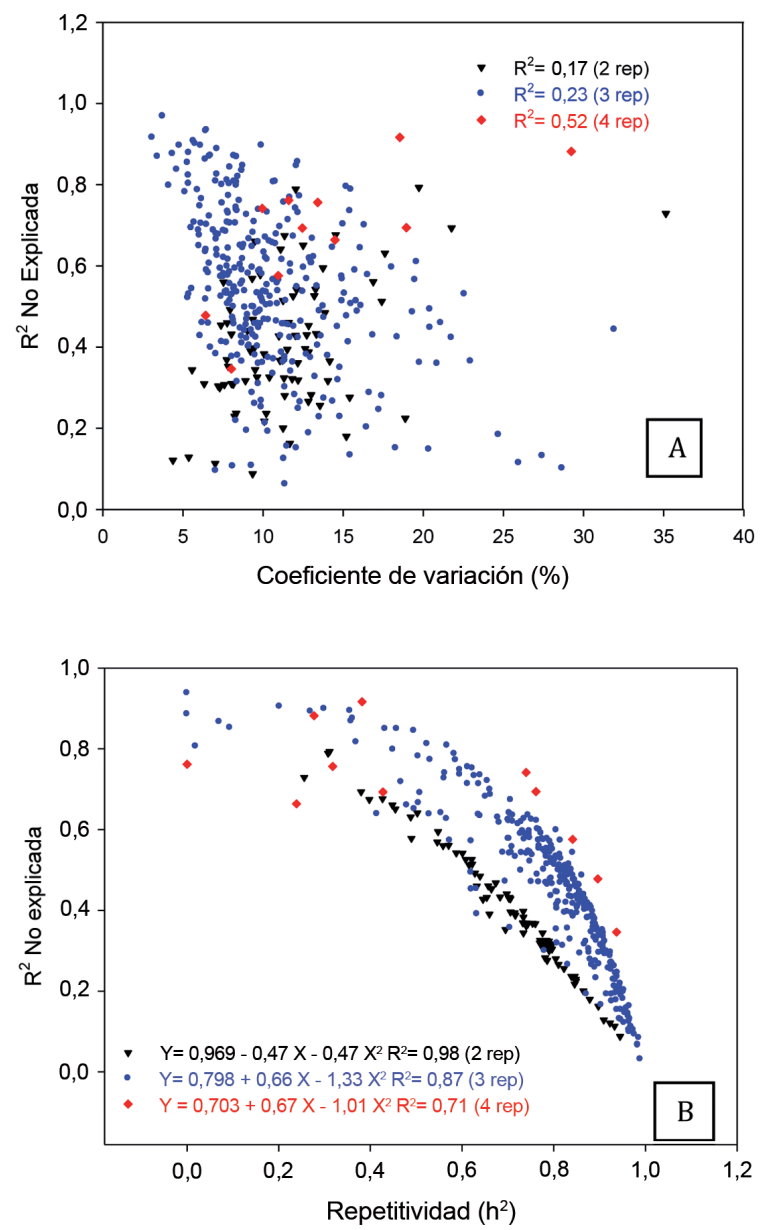

Figura 7. Relación entre el coeficiente de determinación del error o fracción no explicada con el coeficiente de variación y repetitividad, según el número de repeticiones de los experimentos. Panamá. 2000-2013. 
0,71, respectivamente. (Figura 7B). Los resultados de ambos análisis indican claramente, que la repetitividad es un estadístico más relacionado con los componentes de la varianza del modelo que señalan buena precisión experimental, mas no el coeficiente de variación cuya relación depende de la magnitud de la media ambiental del experimento (Yan y Holland, 2010).

Por definición, la precisión experimental está relacionada con la capacidad de encontrar diferencias entre los tratamientos evaluados (Steel y Torrie, 1980). Para estos fines se calculó el cociente entre el estadístico (DMS), para determinar si dos medias son diferentes entre sí y el rango o amplitud de las medias calculada de los tratamientos (DMS/Rango=D/R). En este caso se tomó la DMS por ser el estadístico más sencillo para determinar si dos promedios difieren estadísticamente entre sí, pero bien se pudo tomar el valor calculado por Tukey, Duncan u otras pruebas empleadas para la separación de medias, los cuales dependen del nivel de exigencia que se desea utilizar en cada estudio para separar promedios. Cuando este cociente $(\mathrm{D} / \mathrm{R})$ se acerca a cero $(0,0)$, indica que existen diferencias entre los tratamientos, mientras que cuando el valor de $\mathrm{D} / \mathrm{R}$ se acerca a uno $(1,0)$, prácticamente no hay diferencias entre los tratamientos evaluados y por ende se tuvo una baja precisión experimental. Se relacionó este cociente $(\mathrm{D} / \mathrm{R})$ con los dos coeficientes de determinación que más interesan del modelo $\left(\mathrm{R}^{2}\right.$ de tratamientos y $\mathrm{R}^{2}$ de error) y se encontraron ecuaciones con un $\mathrm{R}^{2}$ de 0,71 y 0,69 para tratamientos y error, respectivamente (Figura 8A y 8B). La relación con la variabilidad de los tratamientos fue inversa, lo que implica que a menor valor del cociente (más precisión experimental) mayor es la variabilidad encontrada entre estos. Mientras que con el error la relación es directa, es decir, a menor valor del cociente menor es la variabilidad no explicada del modelo.

En la Figura 9A y 9B, se presenta la relación del nuevo cociente $(\mathrm{D} / \mathrm{R})$ con los dos estadísticos estudiados $\left(\mathrm{CV} \mathrm{y} \mathrm{h} \mathrm{h}^{2}\right)$ para determinar precisión experimental. Se encontró nuevamente que el coeficiente de variación no tiene una relación robusta con el cociente $\mathrm{D} / \mathrm{R}$, al presentarse mayor dispersión de los datos y un bajo $\mathrm{R}^{2}$ (Figura 9A). Mientras que, el $\mathrm{h}^{2}$ tiene más relación que el CV. Esta relación fue mayor en los ensayos con tres repeticiones que en aquellos que tuvieron dos repeticiones por localidad.
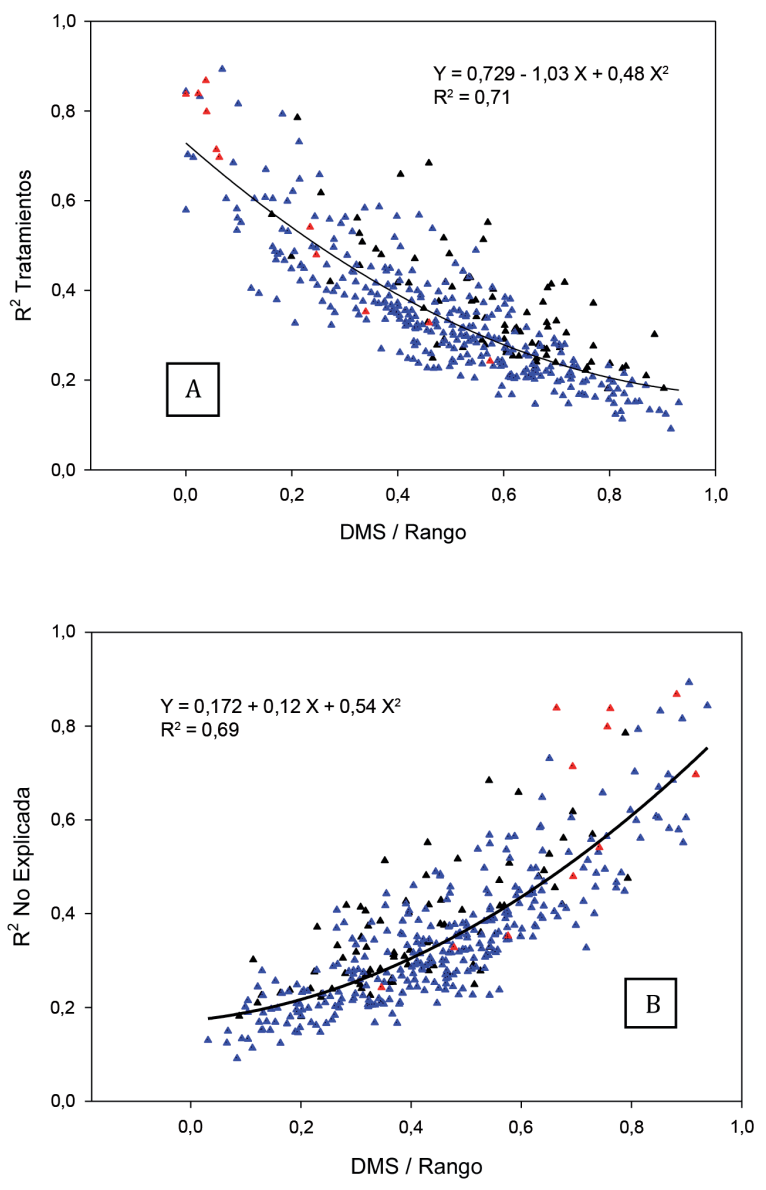

Figura 8. Relación entre el cociente DMS / Rango y la porción atribuida a la diferencia de los tratamientos ( $\mathrm{R}^{2}$ Tratamientos) y la porción no explicada o residuo ( $\mathrm{R}^{2}$ Error). Panamá. 2000-2013.

Los resultados demostraron que el coeficiente de variación está estrechamente asociado con la media general del experimento, relación que le resta confianza como herramienta para medir la precisión experimental, puesto que la media de rendimiento es una variable cuya expresión depende de múltiples factores bióticos y abióticos. Además, se observó que el aumento en el número de repeticiones de los experimentos con igual cuadrado medio del error, se reflejó en una reducción del error estándar, con un aumento de la precisión experimental.

Por otro lado, la repetitividad del experimento presentó baja relación con la media general, no 

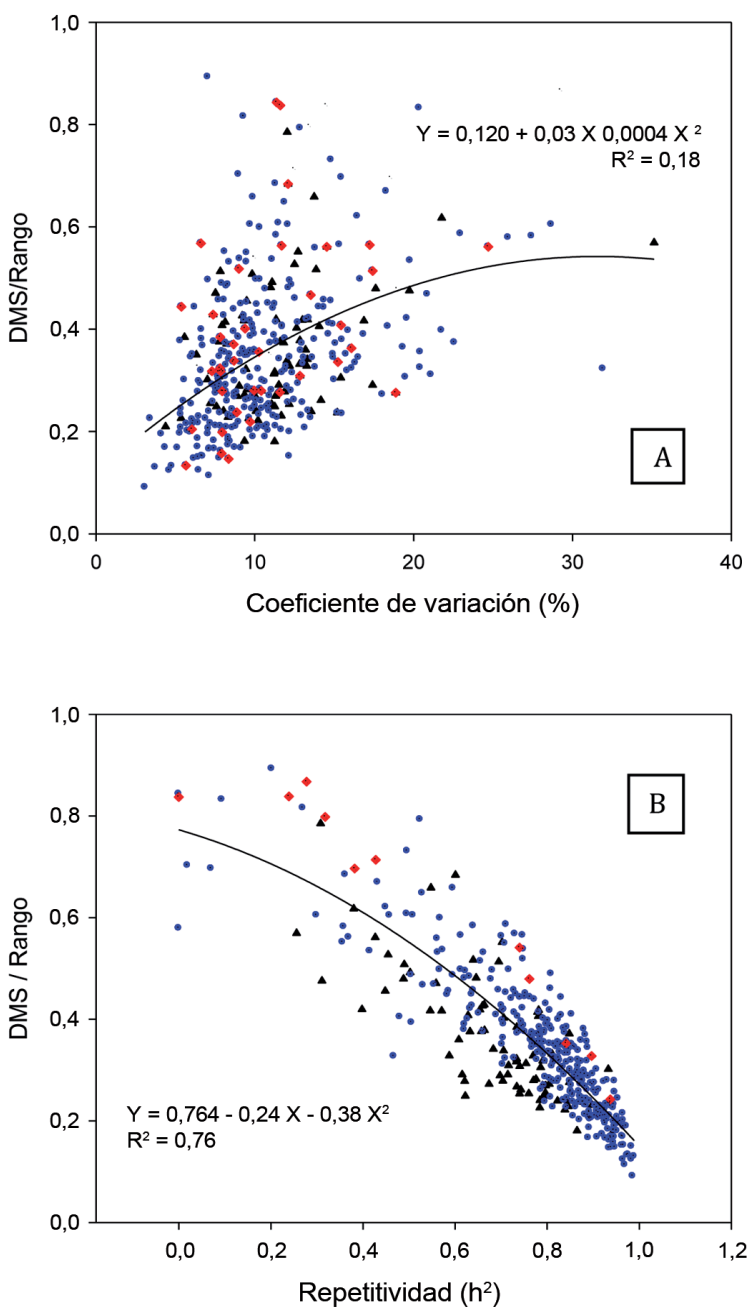

Figura 9. Relación entre el Coeficiente de Variación y Repetitividad con el cociente DMS/Rango (D/R). Panamá. 2000-2013.

obstante, la $\mathrm{h}^{2}$ estuvo altamente relacionada a los $\mathrm{R}^{2}$ tanto de los tratamientos como de la fracción no explicada del modelo. Esta relación denota que la $\mathrm{h}^{2}$ puede ser un estadístico robusto para medir la precisión de los experimentos, con menos sesgo que el CV que depende más de la expresión de la media ambiental.

$\mathrm{Al}$ relacionar ambos estadísticos $\left(\mathrm{CV}\right.$ y $\left.\mathrm{h}^{2}\right)$ con el cociente DMS/Rango se encontró que la $\mathrm{h}^{2}$ está altamente correlacionada con este cociente, mientras que el CV no lo está. En términos generales, estos resultados permiten indicar que la repetitividad es un mejor indicador que el $\mathrm{CV}$, como estimador de buena precisión experimental, debido a que considera en su estimación, la variabilidad por los tratamientos y no la variación del error experimental.

\section{LITERATURA CITADA}

Barreto, H., y W.R. Raun. 1990. La precisión experimental de los ensayos regionales con maíz (Zea mays) a través de Centroamérica. En: T.J. Smyth, W.R. Raun y F. Bertsch, editores, Segundo Taller Latinoamericano de manejo de suelos tropicales, San José, Costa Rica 9-13 julio. Soil Science Department, North Carolina State University, NC, USA. p. 99-105.

Bowman, D.T., y J.O. Rawlings. 1995. Establishing a rejection procedure for crop performance data. Agron. J. 87:147-151.

Bowman, D.T., y C.E. Watson. 1997. Measures of validity in cultivar performance trials. Agron. J. 89:860-866.

Bowman, D.T. 2001. Common use of the CV: a statistical aberration in crop performance trials (Contemporary Issue). J. Cotton Sci. 5:137-141.

Gauch, H.G., y R.W. Zobel. 1996. Optimal replication in selection experiments. Crop Sci. 36:838-843.

Gómez, K.A., y A.A. Gómez 1984. Statistical procedures for agricultural research. $2^{\text {nd }}$ ed. John Wiley Inter Science, Hoboken, NJ, USA.

Martínez, A. 1988. Diseños experimentales: métodos y elementos de teoría. Editorial Trillas, México.

Martínez, E. 2005. Errores frecuentes en la interpretación del coeficiente de determinación lineal. Anuario Jurídico y Económico Escurialense 38:315-332.

Patel, J.K., N.M. Patel, y R.L. Shiyani. 2001. Coefficient of variation in field experiments and yardstick thereof-an empirical study. Curr. Sci. 81(9):1163-1164.

Pimentel, F. 1985. Curso de estatística experimental. Livraria Nobel S.A., São Paulo, Brasil.

Ruíz-Ramírez, J. 2010. Eficiencia relativa y calidad de los experimentos de fertilización en el cultivo de caña de azúcar. Terra Latinoamericana 28:149-154.

Ruíz, D., y A.M. Sánchez. 2006. Apuntes de estadística. www.eumed.net/libros-gratis/2006a/rmss/index.htm. (Consultado 7 jul. 2014).

Silveira Jr., P., J.B. Da Silva, A. Almeida, y E.P. Zonta. 1986. Estatística geral. Estatística Experimental. Universidade Federal de Pelotas, Brasil.

Steel, R.G., y J.H. Torrie. 1980. Principles and procedures of statistics. A biometrical approach. $2^{\text {nd }}$ ed. Mc GrawHill, NY, USA. 
Taylor, S.L., M.E. Payton, y W.R. Raun. 1999. Relationship between mean yield, coefficient of variation, mean square error and plot size in wheat field experiments. Commun. Soil Sci. Plant Anal 30:1439-1447.

Vargas, M., E. Combs, G. Alvarado, G. Atlin, K. Mathews, y J. Crossa. 2013. META: A suite of SAS Programs to analyze multi environment breeding trials. Agron. J. 105:11-19.
Vásquez, E.R., y A. Caballero. 2011. Inconsistencia del coeficiente de variación para expresar la variabilidad de un experimento en un modelo de análisis de varianza. Cultivos Tropicales 32(3):42-45.

Yan, W., y J.B. Holland. 2010. A heritability-adjusted GGE Biplot for test environmental evaluation. Euphytica 171:355-369. 
